Mark Jones

\title{
Angst und Gewalt in der deutschen Revolution von 1918/19
}

Am 18. Januar 1918, etwas mehr als zwei Monate nach dem Ende der Kämpfe des Zweiten Weltkrieges an der Westfront, veröffentlichte die Zeitung "Vorwärts", das wichtigste Organ der SPD, ein Sonderheft anlässlich der - nach Meinung von Zeitgenossen - größten Errungenschaft der im Weltkrieg besiegten deutschen Nation: Am Folgetag würden zum ersten Mal in der deutschen Geschichte alle volljährigen Frauen und Männer das gleiche Recht für die Teilnahme an der Wahl zur verfassungsgebenden Versammlung haben. ${ }^{1}$ Der Sozialdemokrat Erwin Barth beschrieb in dem Artikel dieses Sonderheftes, wie durch das Ausbleiben von Massengewalt während des einwöchigen Ausnahmezustands vor Ende des Krieges die Demokratisierung Deutschlands überhaupt ermöglicht wurde und wie zu Beginn des Monats November das »festeste Militärgebäude der Welt, der scheinbar bestgefügte politische Staatsbau [...] beinahe geräuschlos in sich zusammengesunken [ist]«. Er fügte hinzu:

"Wer hätte sich kurz vorher noch vorstellen können, daß die Revolution in Deutschland so aussehen könnte? Wer hätte geglaubt, daß nicht einer der wortmutigen Generäle mit dem Säbel in der Faust den so oft gepredigten Heldentod für das Kaisertum im Kampfe gegen den Umsturz der alten Ordnung zu suchen wagen würde, und daß gerade die ruhmredigsten Spitzen des preußischen Militärstaats feige und still ins Ausland huschen würden? Es ist alles anders gekommen, als man es sich gedacht hat."«

\footnotetext{
1 Die Literatur zum Zusammenbruch der deutschen Gesellschaft im und nach dem Krieg sowie zur Deutschen Revolution von 1918/19 ist sehr umfangreich. Als Einstieg dazu Jones, Mark: Am Anfang war Gewalt. Die deutsche Revolution 1918/19 und der Beginn der Weimarer Republik, Berlin 2017 sowie Gerwarth, Robert: Die Größte aller Revolutionen. November 1918 und der Aufbruch in eine neue Zeit, München 2018.

2 Barth, Erwin: Die deutsche Revolution, in: Tag der Wahl, Beilage zum Vorwärts, Nr. 31/32, vom 18.01.1919, S. 1f, hier S. 1. Zu den Plänen des Militärs für einen konterrevolutionären Militäreinsatz: Schmidt, Ernst-Heinrich: Heimatheer und Revolution 1918. Die militärischen Gewalten im Heimatgebiet zwischen Oktoberreform und Novemberrevolution (Beiträge zur Militär- und Kriegsgeschichte, Bd. 23), Stuttgart 1981.
} 
Nach über einem Jahrhundert der gemachten historischen Erfahrung, dass Gewalt und Revolution untrennbar gewesen waren, war nun das Ausbleiben von revolutionärer und konterrevolutionärer Gewalt während der deutschen Revolution im November 1918 besonders bemerkenswert. Bereits am 10. November 1918 beschrieb Theodor Wolff, der liberale Herausgeber des »Berliner Tageblatts», das Ende des Kaiserreichs als "größte aller Revolutionen«, denn nie zuvor sei »eine so fest gebaute, mit soliden Mauern umgebene Bastille so in einem Anlauf genommen worden «. ${ }^{3}$ Tags zuvor führte die Zeitung des Finanzbürgertums, der "Berliner Börsen-Courier«, aus, dass die Deutschen nun nicht länger das Wort "Revolution« zu fürchten hätten, da der "wiederauferstandene Geist» von 1848 das "niedergeschlagene» Reich ersetzen würde. ${ }^{4}$ Sogar die ultranationalistische »Tägliche Rundschau« - deren Redakteure wahrlich keine Freunde von Wolff oder des »Vorwärts« waren - kam widerstrebend zu dem Schluss, dass der »am festesten gefügte Staatsbau Europas [...] zusammen[brach] wie ein Kartenhaus, an das die neue Gewalt nur mit dem Finger zu tippen brauchte, um es zum Zusammenbrechen zu bringen«; ein Kommentar, der nicht weit entfernt war von der Analyse der USPD-nahen "Freiheit», in der es hieß: „Wie ein Skelett, das nach langen Jahren der Ruhe aus der Gruft gehoben wird, ist das alte System zusammengebrochen. Es bedurfte kaum eines Stoßes. Ein Lufthauch genügte. ${ }^{5}$ Eine andere sozialdemokratische Zeitung nutzte das Ausbleiben von revolutionärer und konterrevolutionärer Gewalt, um vor allem die deutsche Modernität zu zelebrieren:

"Wo sind sie hin, die alten spießbürgerlichen Träume von Revolution? Die nichts anderes sahen als Mord und Totschlag, als Verwüstung und Vernichtung, als Brand und Plünderei? Alles war in ihren Köpfen nur ein Grauen. In allen früheren revolutionären Umwälzungen ist viel, viel Unheil verübt worden, das nach unseren heutigen Begriffen nicht nötig gewesen wäre. Aber das waren Folgen der Kulturhöhe der damaligen Völker. Es entspricht dem heutigen Stande der Technik und Wirtschaft der Organisations- und Verwaltungswissenschaft, wenn jeder Einzelne bestrebt ist, dem Wohle der Gesamtheit sich unterzuordnen. Die gewaltigste soll zugleich auch die friedlichste und ordnungsgemäßeste Revolution sein. ${ }^{6}$

3 Der Erfolg der Revolution, in: Berliner Tageblatt, Nr. 576 vom 10.11.1918, zit. n. Wolff, Theodor: Tagebücher 1914-1919. Der Erste Weltkrieg und die Entstehung der Weimarer Republik in Tagebüchern, Leitartikeln und Briefen des Chefredakteurs am "Berliner Tageblatt" und Mitbegründers der "Deutschen Demokratischen Partei«, 2 Teile, Boppard am Rhein 1984, S. $814 \mathrm{ff}$.

4 Bundesarchiv Berlin (fortan: BArch Berlin) R901.55577 Bl. 42: 1848-1918, in: Berliner BörsenCourier, Nr. 528 vom 9.11.1918 (Abendausgabe).

5 BArch-Berlin, R901/55625, Bl. 31: Zweihundertvierundzwanzigste Kriegswoche, TR Nr. 590, 18. November 1918 MA; BArch-Berlin, R901/55625, Bl. 42: Es lebe die Freiheit, in: Freiheit, Nr. 1 vom 15.11. 1918.

6 BArch-Berlin, R901/55625, Bl. 39: Die Revolution von heute, in: Chemnitzer Volksstimme, Nr. 267 vom 16.11.1918 MA. Es gab keinen Mangel an militärischen konterrevolutionären Plänen für den Fall 
Wie ist diese optimistische Sicht auf die Zukunft Deutschlands im Winter 1918/ $1919 \mathrm{zu}$ verstehen? Für manche ist diese Sprache Beweis für einen beispiellosen politischen Optimismus in der Zeit kurz nach dem Waffenstillstand. Eine Zeit, in der die Fesseln der Kriegszeit gelöst wurden und die omnipräsente Angst vor dem Tod an der Front verschwand. Eine Zeit, in der mit spontanen Volkstänzen gefeiert wurde, getrieben von einer festlichen Stimmung - das alte Regime war Vergangenheit. Dieses Gefühl findet sich wohl am treffendsten bei Ernst Troeltsch, der Deutschland in der Zeit nach dem Waffenstillstand als "Traumland der Waffenstillstandsperiode» beschrieb - ein Begriff, der nach der Veröffentlichung von Wolfgang Schivelbuschs bahnbrechender Analyse "Die Kultur der Niederlage» weit verbreitete Verwendung erfuhr.

Das folgende Kapitel behandelt das Gegenstück zum optimistischen Diskurs der Zeit: Die Sprache der Krise, die Ausdruck von politischer Angst war. Es soll gezeigt werden, wie die zeitgenössischen politischen Akteure in ihren Diskursen furchtsame Zukunftsszenarien beschworen, um politische Unterstützung zu gewinnen. Anfangspunkt hierfür ist das in Bochum herausgegebene und im ganzen Reichsgebiet vertriebene Organ des Verbandes der Bergarbeiter Deutschlands, die »Bergarbeiter-Zeitung».

Die Krise der revolutionären Imagination: politische Haltung in der "Bergarbeiter-Zeitung" aus Bochum

Die Ausgabe des 9. November 1918, deren Inhalt offensichtlich vor dem Bekanntwerden der bedeutsamen Ereignisse des Tages gedruckt wurde, enthielt bereits Warnungen vor den Gefahren einer Revolution, nämlich die des Bürgerkriegs. Im Zusammenhang mit der Reihe von Reformen, die Historiker*innen in der Retrospektive als "Revolution von oben" beschrieben, vertrat die Zeitung die Meinung, dass die Entlassung Ludendorffs als Chef der Obersten Heeresleitung Ausdruck der neuen Mächteverteilung der politischen Kräfte in Deutschland gewesen sei. Der General wurde auf Geheiß des Reichstags abgesetzt; der Reichstag trieb also die "Beseitigung jeder selbstherrlichen Regierungsgewalt» voran. Das Kommando über die deutsche Armee stand nun unter direkter Kontrolle des Reichskanzlers, somit unter der des Reichstags. Der Reichstag hatte also die Entscheidungsgewalt, über Krieg und Frieden zu entscheiden - ein Recht, was zuvor allein dem Kaiser

einer Revolution in Deutschland, welche zu extremer Gewalt geführt hätten und diese auch kalkuliert gewesen wäre. Dazu Schmidt, Heimatheer und Revolution. 
vorbehalten war. Die verfassungsrechtliche Revolution war in vollem Gange, was sich in den Worten der Zeitung wie folgt niederschlug: "Das ist ein Umsturz von weltgeschichtlicher Tragweite und er ist erfreulicherweise ohne den zeitweilig drohenden blutigen Bürgerkrieg vollzogen worden. «7

Die nächste, nach der Abdankung des Kaisers und dem Triumph der Revolution vom 9. November erschienene Ausgabe der "Bergarbeiter-Zeitung» enthielt eine Bekanntmachung des Vorstands des Verbands der Bergarbeiter Deutschlands. Diese Bekanntmachung war ein Versuch, die Ereignisse der vergangenen zwei Wochen als Teil einer Reihe von positiven Errungenschaften auf dem Weg zur Demokratisierung Deutschlands zu interpretieren:

»Mit elementarer Gewalt setzt sich die Demokratie in Deutschland durch. Die Soldaten vereinigen sich mit den Arbeitern und Bürgern zur Sicherung der demokratischen Freiheiten gegen jeden volksrechtsfeindlichen Versuch, den alten Obrigkeitsstaat wieder herzustellen. Eine gewaltige Bewegung geht durch unser Volk, es sprengt die Fesseln, die es Jahrhunderte gedrückt haben.»

Die Bekanntmachung betont, dass dies die Krönung der Ziele, für die der Verband eintrat, darstellte:

»Bergarbeiter! Unser Verband ist eine demokratische Organisation und kämpft deshalb mit für vollständige Demokratisierung unserer öffentlichen Einrichtungen! Wir standen von jeher im Dienste der Demokratie und stehen darum auch heute mit freudigem Herzen dort, wo die demokratischen Fahnen wehen. Mit unserer ganzen Organisation müssen wir mitkämpften für die Beseitigung alles dessen, was der Volksfreiheit hinderlich ist."

Als Theodor Wolff in seinem berühmten Editorial (»die größte aller Revolutionen«) gerade vor den Gefahren, denen Deutschland gegenüberstand, warnte, umriss der Vorstand des Verbandes der Bergarbeiter Deutschlands die Zukunft des politischen Lebens in Deutschland als Zukunft der existenziellen Krisen: Unabhängig von der Revolution stünde Deutschland ein »harter Winter« bevor; ohne ein großes Ziel vor Augen wäre der Übergang von der Kriegs- zur Friedenszeit unmöglich. Noch schlimmer sei, dass der alte Feind der organisierten Arbeiterklasse noch immer fest im Sattel sitze. Unorganisierte Arbeiter und die, die nach einer radikaleren Revolution strebten, bedrohten die Errungenschaften der Revolution. Ohne die Versorgung durch das Transportnetzwerk würden die Bewohner der deutschen Städte verhungern. Vor dem Hintergrund einer solchen Bedrohung könne es keinen positiven Gründungsmythos geben, der die Errungenschaften der Revolution in der direkten postrevolutionären Zeit legitimiere. ${ }^{8}$

$7 \quad$ O.V.: Heran an die Organisationslosen, in: Bergarbeiter-Zeitung (BAZ), Nr. 45 vom 9.11.1918.

8 O.V.: An die Bergarbeiter, in: BAZ, Nr. 46 vom 16.11.1918. 
Die Zukunftsangst blieb auch in der nächsten Ausgabe präsent. Obwohl die Ausgabe am 23. November 1918 die Neuigkeit der "Erfolge der gewerkschaftlichen Organisation", unter anderem die des Achtstundentags, vermelden konnte, waren die Herausforderungen, vor denen die deutsche wirtschaftliche Entwicklung in der nahen Zukunft stand, zu groß, um das Aufkommen eines positiveren Gründungsmythos zuzulassen:

»Wofür unsere Väter und ihre Söhne 1868, 1872, 1877, 1889, 1905 und 1912 gekämpft und gelitten haben, nun haben wir es erreicht: Achtstundenschicht einschließlich Einund Ausfahrt, Mindestlohn und volle Anerkennung der gewerkschaftlichen Organisation! Wären die sonstigen Verhältnisse in unserem vom Krieg zerfressenen Vaterlande nicht gar so böse, wir könnten eine jubelnde Freude über die Erfolge unserer gewerkschaftlichen Arbeit empfinden. $\aleph^{9}$

Der Artikel machte deutlich, was Deutschland nun bevorstehen würde, warnte vor einer »beispiellos schlimme[n] Zeit» und dass es nur möglich sei, diese zu überwinden, "wenn nun allseitig straffe Selbstzucht, opferwillige Selbstdisziplin geübt wird! Tun wir das nicht, dann sind wir verloren! Dann bricht alles zusammen. Was der Krieg noch verschont hat, wird dann in wahnsinniger Selbstzerfleischung vernichtet!«10

Besonders nachdrücklich warnten dieser und viele weitere Artikel vor dem kommenden Winter, denn ohne Kohle

"[...] können unsere Eisenbahnen wegen Kohlenmangel nicht betrieben werden, dann erhalten wir keine Lebensmittel, eine entsetzliche Hungersnot bricht aus, die Menschen sterben dann massenhaft auf der Straße! Außerdem können wir, wenn keine Kohlen vorhanden sind, auch die Rückführung unsere Soldaten nicht bewerkstelligen, überhaupt die von den Feinden vorgeschriebenen Waffenstillstandsbedingungen nicht erfüllen. Dann aber überfluten die feindlichen Heere unser Land! Wir sind ihnen wehrlos preisgeben!«

Die Ernsthaftigkeit der Lage und was auf dem Spiel stand war mehr als deutlich:

»Bergleute, Kameraden! Wollt ihr, daß die junge deutsche Demokratie infolge innerer Unruhen zusammenbricht? Wollt ihr, daß wir durch lauernde volksrechtsfeindliche Mächte, die dann von ausländischen Soldatenmassen unterstützt werden, wieder in den alten Polizeistaat zurückgeworfen werden? Wollt ihr, daß ein schrecklicher Bürgerkrieg unser unglückliches Volk zerfleischt? «11

Die Sprache des Kriegs wurde in der Nachkriegszeit fortgesetzt. Die Verwendung des Attributs "ausländische Soldatenmassen« ist dabei besonders verblüffend. Dies könnte ein Verweis auf US-amerikanische Soldaten, gleichzeitig auch auf französische Kolonialsoldaten sein - es war ein bekanntes Sprachbild antialliier-

9 O.V.: Erfolg der gewerkschaftlichen Organisation, in: BAZ, Nr. 47 vom 23.11.1918.

10 Ebd.

11 Ebd. 
ter Propaganda während des Ersten Weltkriegs, dessen Verwendung während der Besatzung des Rheinlands und besonders der des Ruhrgebiets vermehrt Verwendung fand.

Die Gefahr des Kohlenmangels war erneut ein zentrales Thema in der letzten Ausgabe des Monats November 1918. Frieden könne nur aufrechterhalten werden, wenn gleichzeitig auch die öffentliche Ordnung bewahrt werden würde. Schon das Einsetzen des ersten Winters nach dem Krieg bedrohte die Ordnung. Einmal mehr wurden die Herausforderungen, denen die deutschen Bergarbeiter gegenüberstanden, als existenzielle Krise dargestellt:

"Aus allen Teilen des Landes kommen schon Hilferufe der Bevölkerung, die in kalten Wohnungen hausen müssen, und die Kälte nimmt zu. Unser armes Volk geht in Hunger und Kälte zugrunde, wenn ihm nicht geholfen wird!

Bergleute! Von euch erwartet das Volk brüderliche Hilfe in dieser Not! Wenn der Bergbau in dieser Schicksalsstunde unseres Volkes nicht voll im Betrieb gehalten wird, dann bricht ein schreckliches Unglück über uns herein! Sind nicht ausreichend Kohlen für unsere Eisenbahnen, Gas- und Elektrizitätswerke, Mühlen, Brotfabriken, für die jetzt umzustellenden Fabriken vorhanden, dann kommt es zu einer Katastrophe, zu einem verwüstenden Durcheinander und die feindlichen Heere ergreifen die diktatorische Gewalt über uns! Mit unserer jungen Freiheit ist es dann vorbei.»

Drei Wochen nach der revolutionären Umformung des Deutschen Kaiserreichs in eine Republik wurden, wie dieser Auszug zeigt, zeitgenössische Erwartungshorizonte von Schreckensvorstellungen eines Zusammenbruchs der Wirtschaft und der sich daran anschließenden Revision der politischen Errungenschaften der Revolution überlagert.

Zu Beginn des Monats Dezember 1918 griff auch Karl Legien die zuvor beschworene Krise der Erwartungen in seinem Artikel »Mehr Kohlen «12 auf. Erneut wurde betont, die Versorgung mit Kohle sei für die deutsche Wirtschaft existenziell; ohne sie stünde Deutschland am Abgrund. Legien verteidigte im Folgenden den Ruf der Bergarbeiter und der Kohleindustrie gegenüber dem Vorwurf, der Industriezweig mit seinen Beschäftigten sei verantwortlich für die verschlechterte Versorgungslage seit der Revolution und dem Waffenstillstand. Legien wies darauf hin, dass der plötzliche Einbruch der Versorgung in diesem äußert kritischen Moment primär durch den heftigen Rückgang der Arbeitskräfte verursacht wurde: Durch den Waffenstillstand gingen der deutschen Wirtschaft die Kriegsgefangenen verloren, die zu Kriegszeiten bis zu 20 Prozent der Arbeitskräfte in der Kohleindustrie stellten. Die tatsächliche Zahl der Kriegsgefangenen, die die deutschen Bergwerke als Konsequenz des Waffenstillstands verließen, belief sich auf 162085 .

Legien, Karl: Mehr Kohlen, in: BAZ, Nr. 50 vom 14.12.1918. 
Vor dem Hintergrund des dramatischen Abgangs der Arbeitskräfte wies Legien eindringlich darauf hin, dass es nicht die Zeit für Streiks sei. Er appellierte an die Leser*innen:

»Duldet keine wilden Streiks, womit jetzt unser eigenes Porzellan zerschlagen wird! Alle Streikfragen können und müssen jetzt auf dem Verhandlungswege erledigt werden. Hilf mit, Hunger und Arbeitslosigkeit abzuwehren, den völligen Zusammenbruch unserer Volkswirtschaft zu verhindern und unsere Zukunft zu sichern durch mehr Kohlen!«

Von diesem Punkt ausgehend erklärte Legien das Ergebnis der jüngsten Verhandlungen (das bekannte Stinnes-Legien-Abkommen) und bat eindringlich um die Fortsetzung der Kohleförderung sowie den Verzicht auf Streik:

»Man muss berücksichtigen, in welcher entsetzlichen Lage sich Deutschland und seine Bevölkerung nach diesem Ausgang des Krieges befindet. Auch dann, wenn die Revolution nicht im Anschluß an die vernichtenden Wirkungen des Krieges eingetreten wäre, müßte durch ungestörten Fortgang der Produktion ihr Erfolg gesichert werden, denn nichts könnte diesen mehr gefährden, als Arbeitslosigkeit und Not in der Bevölkerung. Unabwägbar aber wäre die Wirkung einer solchen Notlage unter den jammervollen Wirtschaftsverhältnissen, die über Deutschland durch den Krieg hereingebrochen sind. Dem muß vorgebeugt werden. «13

Mitte Dezember 1918, als die revolutionär-politische Krise, die sich in Berlin manifestiert hatte, und die Aktivitäten der Spartakisten im Ruhrgebiet offensichtlicher wurden, färbte sich die Sprache der Presse immer mehr in Richtung einer unmittelbar bevorstehenden Katastrophe. Am 25. Dezember 1918, inmitten der ersten gewalttätigen revolutionären Krise in der deutschen Hauptstadt, schrieb der einflussreiche Journalist Alexander Redlich, dass Deutschland »am Rande des Abgrunds« sei. Am selben Tag, stellvertretend für die Art und Weise, wie diese Debatte im öffentlichen Raum stand, schrieb der Historiker Gustav Mayer: „Es geht dem Abgrund zu! Der Schlund der Hölle hat sich aufgetan. Deutschland versinkt. Ob das andere Europa es um vieles überleben wird, wer vermöchte das heute zu sagen! Die Ereignisse des gestrigen Tages machen mich ganz mutlos. «14

Vier Tage, bevor diese Meinung in- und außerhalb Berlins die Runde machte, war sie bereits in einem Abriss der Ereignisse in der "Bergarbeiter-Zeitung" zu lesen. Auf einer außergewöhnlichen Titelseite vom 21. Dezember 1918 warnte das Organ des Verbandes der Bergarbeiter Deutschlands, dass Deutschland "vor dem Abgrund « sei.

\footnotetext{
13 Ebd.

14 Mayer, Gustav: Als deutsch-jüdischer Historiker in Krieg und Revolution 1914-1920. Tagebücher, Aufzeichnungen, Briefe (Deutsche Geschichtsquellen des 19. und 20. Jahrhunderts, Bd. 65), hier 25. Dezember 1918, S. 203. Am 26. Dezember nutzte der Historiker Karl Hampe denselben Ausdruck - Abgrund - in seinem Tagebuch. Hampe, Karl: Kriegstagebuch 1914-1919 (Deutsche Geschichtsquellen des 19. und 20. Jahrhunderts, Bd. 63), München ${ }^{2} 2007$, 26. Dezember 1918, 27. Dezember 1918, S. 807.
} 
„Das `Land der Organisation` bietet jetzt nicht durchweg ein erhebendes Schauspiel von Selbstbesinnung. Das köstliche Gut der Freiheit wird von den Vielen, die sich allzusehr an die Polizeisuchtel gewöhnten, nicht gewürdigt. Die provisorische Regierung der sozialistischen Volksbeauftragten (Ebert-Haase) kann sich zweifellos auf eine ungeheure Volksmehrheit stützen, jedoch in zahlreichen Orten etablierten plötzlich aus dem Dunkel auftauchende diktatorische Emporkömmlinge eine „,Republik für sich: Unter der Führung eines jeder Selbstdisziplin baren ıSpartakus` wird zunächst die Berliner Bevölkerung frivol beunruhigt. Mit Maschinengewehren ausgerüstete kleine Banden suchen die Volksregierung zu terrorisieren, die demokratische Neuordnung durch die Nationalversammlung gewaltsam zu hintertreiben. Die siegestrunkenen Militärs der Entente machen sich bereit, ihrerseits diktatorische `Ordnung in dem unglücklichen Deutschland zu schaffen. Geborene Feinde der Demokratie und einer deutlichen Einheitsrepublik benutzen die spartakistischen und ähnlichen Wirrnisse, um Deutschland zu zerreißen in wer weiß wie viele Kleinstaaten, deren politische und wirtschaftliche Ohnmacht auf der Hand liegt. Die Arbeiterschaft will ein einheitliches deutsches Reichs- und Wirtschaftsgebiet, weil wir nur in einem solchen die ungeheuerlichen Kriegsschäden mit ernster Gemeinschaftsarbeit überwinden können."

Die Verbindungen zwischen der Gewalt des Ersten Weltkriegs, versinnbildlicht in dem Maschinengewehr, und der Bedrohung für die demokratische Zukunft Deutschlands wurden in einer langen Passage am deutlichsten, in der nicht weniger als sechs Mal das Maschinengewehr als Referenz auftauchte:

„Mit Maschinengewehren soll die gesetzliche Neuordnung Deutschlands verhindert werden, mit Maschinengewehren wird dort die demokratische Meinungs- und Preßfreiheit unterdrückt, mit Maschinengewehren soll die ,Sozialisierung der Betriebe' erzwungen werden, mit Maschinengewehren werden Betriebsleiter zu Handlungen gegen ihre Überzeugung gezwungen, mit Maschinengewehren dringt man in die Wohnungen sozialistischer Minister und Parteiführer ein, um sie zur Abdankung zu zwingen, mit Maschinengewehren geht man vor gegen gewerkschaftliche Organisationen, sucht sie zu zerreißen, ihre Verträge zu durchbrechen, der Disziplinlosigkeit, der Anarchie zum Triumph zu verhelfen. Und das alles geschieht von einer kleinen, allerdings lärmstarken und gewaltfrechen Minderheit, deren zahlenmäßige Bedeutungslosigkeit sich schon durch die Arbeiterund Soldatenrats-Wahlen in Dresden und Chemnitz klipp und klar herausgestellt hat. Diese Maschinengewehrpolitik ist ein Hohn auf die Demokratie! « ${ }^{15}$

Bereits am 9. Dezember 1918 verwendete die "Vossische Zeitung» für die Tagespolitik den Ausdruck "Maschinengewehrpolitik«16. Eben dieser Ausdruck hatte im Verlauf des Monats November an medialer Popularität gewonnen, als sich die Sprache der politischen Mobilisierung ähnlich intensivierte wie die auf den Straßen Berlins teils gewaltsam ausgetragene Politik (besonders am 6. und am 24. Dezember). Bemerkenswert ist, dass sich die Sprache eines reichsweit erhältlichen gewerkschaftlichen Blattes wie der "Bergarbeiter-Zeitung" nicht sonderlich von der ihres Berliner Pendants unterschied - es lässt sich vermuten, dass die Erfahrung der Angst vor Gewalt während der Revolution von 1918/19 nicht regional

15 O.V.: Vor dem Abgrund, in: BAZ, Nr. 51 vom 21.12.1918.

16 O.V.: Maschinengewehr-Politik, in: Vossische Zeitung, Nr. 629 vom 9.12.1918 (Abendausgabe). 
konzentriert und ausgeprägt war, wie viele Historiker*innen womöglich fälschlicherweise annehmen.

In der ersten Ausgabe des neuen Jahres 1919 gab die »Bergarbeiter-Zeitung» Berichte aus anderen Quellen darüber wieder, dass die Revolution die alte Ordnung beseitigt habe:

»Nach mehr als vier Jahren beispielloser Zerstörung hat uns das vergangene Jahr einen Zusammenbruch gebracht, dessen Umfang auch nicht annährend zu ermessen ist. Die alten Gewalten: Absolutismus, Bureaukratismus und Militarismus wurden in den Strudel dieses Zusammenbruchs mit hinabgerissen. Was für Ewigkeiten festgefügt schien, wurde von Minuten weggefegt. Aller mittelalterlich romantisch-reaktionäre Wust und Unrat versanken im Orkus. Wir müssen uns nun eine neue, bessere Ordnung fügen, neugestalten, was morsch und faul war und dem Sturm der Zeit nicht standhalten konnte. «17

Mit der Festlegung der Wahltage für die Nationalversammlung und die verfassungsgebende preußische Landesversammlung auf den 19. bzw. 26. Januar wurde die Bedeutung dieser kommenden Wahlen betont.

»Noch nie hat ein Volk vor einer so schwerwiegenden Entscheidung gestanden, wie jetzt das deutsche. Der Wahltag ist diesmal mehr als je Zahltag. Es muß abgerechnet werden mit den gewissenlosen Blutsaugern, Kriegstreibern, Kriegsgewinnlern, Schiebern, Wucherern usw. Alle diesem Diebes- und Lumpengesindel muß am Wahltag das Handwerk dauernd gelegt werden. «18 $^{18}$

Die hier verwendete Sprache suggeriert, es hätte dank der Revolution einen Umbruch gegeben, und das vorherige System könne nun durch etwas Besseres ersetzt werden, ergo würde der neuen Republik gegenüber ein positiver Grundtenor entstehen. Direkt daran anschließend fokussierte sich die Sprache erneut auf die unmittelbar bevorstehenden Gefahren. Als die "Bergarbeiter-Zeitung» am 4. Januar 1919 die positiven Aspekte der Gründung der Weimarer Republik umriss, warnte sie nichtsdestotrotz:

"Am Wahltag werden die Augen der ganzen Welt auf uns gerichtet sein, weil es nicht nur um das Schicksal der deutschen, sondern mehr oder weniger auch um das der Arbeiter aller Länder geht. Nur ein reiner Volkssieg verbürgt die Errungenschaften der Revolution und bringt uns dem angestrebten Rechtsfrieden näher. Die Hoffnungen der Arbeiter und Friedensfreunde aller Länder beruhen auf einem Volkssieg. Ein Volkssieg wäre die größte und schönste Tat im neuen Jahre. «19

Die Suggestion einer konstanten Bedrohung war in der nächsten Ausgabe vom 11. Januar 1919, am Tag der Beendigung der spartakistischen Besetzung der Redaktion des »Vorwärts» durch das Freikorps Reinhard, sogar noch stärker. Die 
Sprache der Mobilisierung hatte eine negative Färbung und warnte vor dem, was vermieden werden müsse, anstatt die Vorteile, die die Revolution gebracht hatte und bringen könnte, in den Vordergrund zu stellen:20

»Die deutsche Nationalversammlung aber ist berufen, uns vor fremdländischer Vergewaltigung zu beschützen, über die uns zugedachte Friedensbedingungen zu beschließen. Eine verantwortungsreiche Aufgabe. Die Phantasten und Wahnwitzigen, die hofften und Leichtgläubigen versicherten, einem besiegten Deutschland würden die ausländischen Friedensvorkämpfer und Proletariermassen beistehen gegen unerträglichen Demütigung und Belastung, können sich jetzt davon überzeugen, daß sie sich selbst täuschten und betrogen. Wo sind jetzt die erwarteten ausländischen Helfer? Präsident Wilson selbst, der für einen Rechtsfrieden und versöhnlichen Völkerbund eintritt, wird von den siegberauschten annexions- und gelddurstigen Kriegspolitikern in den Ententestaaten bekämpft; und er hat im eigenen Lande keine Parlamentsmehrheit hinter sich. In England haben vor kurzem die Parlamentswahlen mit einer Niederlage aller hervorragenden Vertreter des Versöhnungsfriedens geendet! In Frankreich beherrschen die Rache und Annexionslust die öffentliche Meinung. In Belgien und Italien ist es nichts anders. ¿21 $^{21}$

Die Zeitung erinnerte daran, dass Deutschland den Friedensvertrag von Versailles als Verlierer des Weltkriegs unterzeichnet hatte und gleichzeitig die Wähler sicherstellen müssten, dass es zu keiner Diktatur, weder von links noch von rechts, käme. Der 19. Januar 1919 war ein Tag, an dem sich entscheiden sollte, »ob das neue Deutschland eine demokratisch verwaltete Republik sein soll oder nicht. [...] Niemals war unser gesamtes Volk vor eine so unabsehbar bedeutungsvolle Entscheidung gestellt wie am 19. Januar 1919!«22

Im Verlaufe des Monats Februar 1919, während eine Welle von Streiks über Deutschland schwappte, legte die »Bergarbeiter-Zeitung» ihren Fokus auf die Bedrohung durch den Anarchismus, die wilden Streiks und vor allem den Spartakismus. ${ }^{23}$ Am 1. März schrieb die "Bergarbeiter-Zeitung" - ähnlich wie die gesamte deutsche Presse, die mit ihrer Wortwahl das nahende politische Armageddon suggerierte - nun vom "Spartakusterror» und warnte vor dem möglichen Sieg der Spartakisten. ${ }^{24}$ In einem langen Artikel, der die Verbrechen der Spartakisten heraushob, rief die Zeitung die Arbeiter dazu auf, sich nicht an Streiks zu beteiligen, und verteidigte die Regierung in der Ansicht, dass eine Bewaffnung der Arbeiterschicht - und somit eine proletarische Revolution - nicht nötig sei:

"So kann es nicht weiter gehen! Dieser Ruf wird immer lauter von den in ihrer Freiheit vergewaltigten Arbeitern erhoben. Stellenweise haben sie zur Selbsthilfe gegriffen. Es ist zu schweren Zusammenstößen gekommen, wobei Arbeiterblut geflossen ist. Das kann in

O.V.: Bergarbeitermassen, marschiert auf, in: BAZ, Nr. 2 vom 11.1.1919.

Ebd.

Ebd. Zu den gemischten Reaktionen auf die Wahlergebnisse: O.V.: Frei im Volkstaat, in: BAZ, Nr. 5 vom 1.2.1919.

23 O.V.: Wie Spartakus schimpft und verleumdet, in: BAZ, Nr. 8 vom 22.2.1919.

24 Ebd. 
der Tat so nicht weiter gehen. Unter allen Umständen und mit allen Mitteln muß dafür gesorgt werden, daß die Freiheit und Sicherheit der Arbeiter in jeder Beziehung gewährleistet sind. Der Spartakusterror soll und darf nicht triumphieren. Das wäre Selbstmord und Wahnsinn. «25

Auch am 8. März 1919 war die Sprache der Mobilisierung beständig gegen die Spartakisten gerichtet, welche an dieser Stelle stellvertretend für alle Gruppen standen, die sich an Streiks beteiligten. Für die Streikführer war es essentiell, dem (sowjet)russischen Beispiel in punkto Revolution zu folgen; darauf ausgerichtet war die Rhetorik ihrer politischen Gegner: "In endlosen Reden wurde gewettert gegen die Gewerkschaftsführer, die Scheidemänner, die Mehrheitssozialisten, die Blutdiktatur Ebert-Scheidemann-Noske, die Mörder Liebknechts und Rosa Luxemburgs, den Militarismus, den weißen Schrecken usw. Aber an der Sozialisierung selbst wurde nicht gearbeitet. ${ }^{26}$

Nach den Streiks der Monate November und Dezember warnte die »Bergarbeiter-Zeitung«, die Fortsetzung der Streiks würde der Arbeiterklasse am meisten schaden. Diese Streiks würden stattfinden, "um die Demokratie zu vernichten". Sie wären nur durch die »Waffengewalt» der Gegner der Demokratie durchsetzbar, mit deren Hilfe die Streikführer die Mehrheit der Arbeiter gegen ihren Willen zu Streiks zwängen. In den Worten der »Bergarbeiter-Zeitung« hieß es:

"Das ist Autokratie und Gewalttätigkeit in höchster Vollendung. Dagegen wehren wir uns, schon weil wir Demokraten sind, denen des Volkes Wille das höchste Gesetz ist. Wir wehren uns aber auch dagegen, weil die Arbeiter durch diese Streiks sich selbst aushungern, dazu noch ihren Lohn opfern und zum Totengräber ihrer Freiheit werden. [...] Nicht durch Autokratie, sondern nur durch Demokratie können wir zum Sozialismus kommen. ${ }^{27}$

Eine Woche später, nach Ende der Berliner Märzkämpfe, druckte die »Bergarbeiter-Zeitung« eine Erklärung der Reichsregierung ab und zeigte damit, dass die Zeitung auf der Seite der Regierung stand:

»Wilde Sozialisierungsversuche aber, terroristischen Zwang gegen die Arbeiterschaft, bewaffneten Aufstand, Zerstückelung des Reichs werden wir rücksichtslos bekämpfen. Uns ist jedes Menschenleben heilig. Die Revolution gibt keinen Freibrief auf Raub, Mord und Gewalttätigkeit jeder Art. Über allem steht das Leben des Volkes. Wer sich an ihm vergeht, ist unser Feind! Die Strenge des Gesetzes wird ihn treffen! Nach vier Jahren furchtbaren Krieges mit ungeheuren Zerstörungen an Kulturwerten und einem Meer von Blut wollen wir nicht, daß auch noch die Schrecknisse des Bürgerkriegs mit seinen mörderischen Bruderkämpfen, mit all seinem Haß und seiner Zerrüttung unser Vaterland zerstören. [...] Eine gewaltige Mehrheit von 22 Millionen Wählern hat uns zur Reichsregierung berufen. Steht zu uns, wie wir zu euch stehen! Das ganze Volk schließt sich uns an gegen 
Vergewaltigung, Zerstörung, Zusammenbruch. Wenn wir einig sind, ist uns die Zukunft sicher!«28

Ein solches Zitat zeigt, wie die reichsweite gewerkschaftliche Presse die Sprache der Mobilisierung, die von Weimar ausging, aufgriff. Im Februar und März 1919 erreichte die durch die andauernden Streiks und Ausbrüche von Gewalt geprägte Phase der Revolution ein Stadium, das Detlef Peukert als »Zeit der Enttäuschungen« beschrieb. Die medialen Diskurse, die die ersten Errungenschaften der Revolution positiv betrachteten, waren nun längst vergessen. Die Kombination aus wirtschaftlichem Elend, militärischer Niederlage, Zukunftsangst und politischen Grabenkämpfen zwischen linken Gruppierungen war zu groß, um einen positiven Mythos der Gründung der Republik entstehen zu lassen. All das, was in dieser Phase von der Revolution übrigblieb, waren Zukunftsängste und die Bedrohung durch "russische Verhältnisse» in Deutschland. Ein Anti-Chaos-Reflex dominierte die Sprache der politischen Mobilisierung auf Kosten von optimistischeren Diskursen über die Gründung der Republik.

\section{Deutschland und die "Shatter-Zones» der untergegangenen Monarchien}

Die Perspektive der »Bergarbeiter-Zeitung» kann nur voll und ganz verstanden werden, wenn die Herausforderungen, vor denen die Anführer der organisierten Bergarbeiter standen, im Kontext einer grundsätzlich bedrohlichen transnationalen Umgebung gesehen und berücksichtigt werden. Im Winter 1918/19 waren die deutschen Zukunftsvorstellungen stark geprägt vom rasanten Aufstieg politischer Gewalt in weiten Teilen Zentral-, Ost- und Südosteuropas, die auf die Zerschlagung der Monarchien im zaristischen Russland, Österreich-Ungarn und dem Osmanischen Reich folgte. ${ }^{29}$

Die deutsche Antwort auf die Angst vor chaotischen Zuständen war staatliche Gewalt um der Ordnung willen. Sie war der Schild, der die Zeitgenossen vor "russischen Verhältnissen« und ihrer tiefsten Angst davor schützte. Auch auf internationaler Ebene wurde mit der Ausübung von Gewalt ein Zeichen gesetzt: Während die Friedensverträge in Paris ausgearbeitet wurden, zeigte der neue deutsche Staat den verbliebenen Mächten, dass er stark genug sei, um den Bedrohungen aus dem Osten ohne weitere Interventionen Großbritanniens und Frankreichs zu

28 O.V.: An das deutsche Volk, in: BAZ, Nr. 11 vom 15.03.1919.

29 Gerwarth, Robert/ Horne, John (Hrsg.): War in Peace. Paramilitary Violence in Europe after the Great War (The greater war), Oxford 2012; Horne, John: Introduction, in: A Companion to World War I, hrsg. von dems., Oxford 2010, S. XVI. 
trotzen. Wie die Auszüge aus der politischen Presse deutlich zeigen, beeinflusste diese Attitüde die Haltung der deutschen Bevölkerung hinsichtlich der Anwendung von Gewalt durch den Staat und erklärt, warum so viele Deutsche bereit waren, den Staat bei der Ausübung von Gewalt zu unterstützen. ${ }^{30}$ Immer wieder wurde in dieser Situation angeführt, dass die siegreiche Entente ohne eine Machtdemonstration des deutschen Staates ihre Besatzung von deutschem Staatsterritorium weit über das Rheinland ausweiten würde. ${ }^{31}$

\section{Ausübung der Gewalt}

Zu den Höhepunkten der gewaltsamen Ausübung von staatlicher Autorität kam es in Berlin und München im März und Mai 1919. Am 3. März 1919 erklärte ein Zusammenschluss sozialistischer Gruppen in Berlin, unter anderem die Berliner SPD - Eberts und Noskes Parteigenossen -, die USPD und die Spartakisten, dass sich die Arbeiter*innen der Stadt der Streikwelle, die Mittel- und Westdeutschland im Verlauf des Monats Februar erfasst hatte, anschließen würden. ${ }^{32}$ Die Forderungen, mit denen der Streik begründet wurde, reichten von der Beseitigung der noch immer kaiserlichen Militärrechtsprechung (die synonym mit dem Schutz der Mörder Liebknechts und Luxemburg gesehen wurde) bis hin zur vollständigen Übernahme der Macht im Staat nach bolschewistischem Vorbild. Gustav Noske fuhr als Antwort eine harte Linie; Bürgerrechte wurden ausgesetzt und 30000 Freikorpssoldaten unter dem Kommando von General Lüttwitz zur Bekämpfung des Streiks entsandt. ${ }^{33}$

Diese militärische Präsenz führte kurze Zeit später zu einer rapiden Eskalation der Gewalt. Zunächst wurden die ersten Randalierer und Plünderer beschuldigt, Soldaten und Offiziere in der Nähe des Alexanderplatzes angegriffen zu haben;

\footnotetext{
30 Jones, Am Anfang war Gewalt, S. 180.

31 Das Tagebuch des Geschichtsprofessors Karl Hampe zeigt die Bedeutung der Ängste vor einer alliierten Invasion.

32 Zum Verlauf des Streiks und der Revolutionspolitik 1918-1919: Kolb, Eberhard: Die Arbeiterräte in der deutschen Innenpolitik 1918 - 1919, Frankfurt a. M. 1978; Kluge, Ulrich: Soldatenräte und Revolution. Studien zur Militärpolitik in Deutschland 1918/19 (Kritische Studien zur Geschichtswissenschaft, Bd. 14), Göttingen 1975; Kluge, Ulrich: Die deutsche Revolution: 1918/1919. Staat, Politik und Gesellschaft zwischen Weltkrieg und Kapp-Putsch (Moderne deutsche Geschichte, Bd. 8), Frankfurt a. M. 1997; Morgan, David W.: The Socialist Left and the German Revolution. A history of the German Independent Social Democratic Party 1917-1922, Ithaca/ New York/ London 1975; Miller, Susanne: Die Bürde der Macht. Die deutsche Sozialdemokratie 1918-1920 (Beiträge zur Geschichte des Parlamentarismus und der politischen Parteien, Bd. 63), Düsseldorf 1978; Winkler, Heinrich August: Von der Revolution zur Stabilisierung. Arbeiter und Arbeiterbewegung in der Weimarer Republik 1918 bis 1924(Geschichte der Arbeiter und der Arbeiterbewegung in Deutschland seit dem Ende des 18. Jahrhunderts, Bd. 9), Berlin ${ }^{2} 1985$.

33 Wette, Wolfram: Gustav Noske. Eine politische Biographie, Düsseldorf ${ }^{2} 1988$, S. 416.
} 
daraus entwickelte sich in der Nacht vom 5. auf den 6. März am Polizeipräsidium Alexanderplatz eine ausgewachsene Schlacht unter Einsatz von Artillerie und schweren Maschinengewehren. In der Stadt standen sich unterschiedliche bewaffnete Gruppen gegenüber, unter anderem die aufständischen Matrosen, die während der Weihnachtskämpfe noch siegreich waren, sowie Mitglieder der regierungstreuen Sicherheitspolizei, einer paramilitärischen Einheit der Polizei zur Bekämpfung von Aufständen, und Freikorpstruppen unter dem Kommando von Lüttwitz. ${ }^{34}$ Jede der Parteien beschuldigte die jeweils anderen, Schuld an der Eskalation der Situation zu sein. ${ }^{35}$ Unterstützer der Regierung argumentierten, dass die sozialrevolutionären Aufständischen im Zuge der Durchführung eines Fünftagesplans zur Machtübernahme und zur Gründung einer deutschen Sowjetrepublik zu den Waffen gegriffen hätten. ${ }^{36}$ Wie es bei eskalierender Gewalt häufig der Fall ist, beschuldigten sich beide Seiten gegenseitig, Gräueltaten begangen zu haben. ${ }^{37}$ Während sich die Kämpfe in Berlin ausbreiteten, gewannen regierungstreue Truppen bald die Oberhand. Nachdem die Freikorpssoldaten den Alexanderplatz unter ihre Kontrolle brachten, brach der Streik, der nur widerwillig von der Berliner Arbeiterschaft unterstützt wurde, am 7. März zusammen. Am nächsten Tag notierte Harry Kessler in sein Tagebuch, dass »in der Reichskanzlei [...] ein Siegesrausch [herrsche]. Der Himmel hänge den Mehrheitssozialisten voller Geigen. Alle Schwierigkeiten schienen ihnen behoben, weil sie mit Hilfe Reinhardts

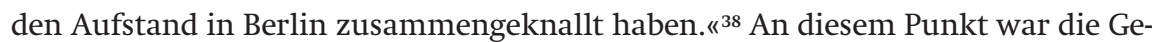
walt allerdings erst an ihrem Anfang. ${ }^{39}$

Eine unbekannte Anzahl von Aufständischen bewegte sich nach der Niederlage am Alexanderplatz in Richtung der Arbeiterviertel in Ostberlin, wo sie sich erneut verbarrikadierten. Regierungstruppen versuchten, die neuen Hochburgen zu umstellen und sie zu zerstören. Dies wurde durch die Feuerkraft, die den Freikorps zur Verfügung stand und den Aufständischen weit überlegen war, ermöglicht: Die Gebiete, in denen Regierungstruppen aufständische Kämpfer vermuteten, wurden mit Artillerie und Maschinengewehren sowie Fliegerbomben angegriffen.

34 Zu den Ursprüngen der Republikanischen Sicherheitswehr und der Volksmarinedivision: Stephenson, Scott: The Final Battle. Soldiers of the Western Front and the German Revolution of 1918 (Studies in the social and cultural history of modern warfare, Bd. 30), Cambridge 2009, S. 261-265.

35 Jones, Am Anfang war Gewalt, S. 238-248.

36 Ebd. Agents provocateurs könnten tatsächlich das Feuer eröffnet haben, dazu die Anschuldigungen in: USPD (Hrsg.): Die Wahrheit über die Berliner Straßenkämpfe, Berlin 1919, S. 6; Müller, Richard: Der Bürgerkrieg in Deutschland. Geburtswehen der Republik, Berlin 1925, S. $170 \mathrm{f}$.

37 O.V.: Wer hat begonnen? Die ersten Straßenschlachten, in: Vorwärts, Nr. 126 vom 10.3.1919 (Morgenausgabe); O.V.: Die Straßenkämpfe in Berlin, in: Die Freiheit, Nr. 114 vom 9.3.1919 (Morgenausgabe).

38 Kessler, Charles: The Diaries of a Cosmopolitan 1918-1937, Count Harry Kessler. Translated and edited by Charles Kessler, London 1999, S. 83 (8.3.1919).

39 Jones Am Anfang war Gewalt, S. $250-275$. 
Da diese Gebiete stark besiedelt waren, gingen die Opferzahlen in die Höhe. ${ }^{40}$ Als am 12. März die Kämpfe ein Ende fanden, waren mindestens 1.200 Menschen tot, von denen nur 75 Angehörige der regierungstreuen Truppen waren. ${ }^{41}$ Eine dreistellige Zahl von Verletzten wurde in Krankenhäuser eingeliefert.42 Als düsterer Ausdruck der Faszination der Öffentlichkeit gegenüber den Auswirkungen des Einsatzes von Kriegsgerät auf den menschlichen Körper druckten die Zeitungen Aufrufe ab, dass sich »Kriegstouristen» von den überfüllten städtischen Leichenhallen fernhalten sollten, sofern sie nicht ernsthaft nach vermissten Verwandten suchten. ${ }^{43}$

\section{Gewalt und Wiederaufbau}

Weimar war nicht der einzige Staat, dessen Herrschaftsanspruch und Legitimation sich auf die (Möglichkeit der) Ausübung von Gewalt stützte. In seiner Arbeit über Frankreich des 19. Jahrhunderts nutzte der Historiker Robert Tombs den Begriff des »Gründungsmassakers«, um damit der exzessiven Gewalt des April 1832, Juni 1848, Dezember 1851 und Mai 1871 Ausdruck zu verleihen. ${ }^{44}$ Wie eine Untersuchung des transnationalen Kontexts der imperialen Desintegration nach dem Ersten Weltkrieg zeigt, begingen eine Reihe von Staaten neben Deutschland „Gründungsmassaker» der einen oder anderen Art. Beispiele dafür sind der ungarische "weiße Terror" nach dem Zusammenbruch der ungarischen kommunistischen Republik, diverse Ausbrüche von Gewalt während des griechisch-türkischen Krieges oder die Adaption der sorder to executer im Irischen Freistaat, der zwar Ähnlichkeiten mit dem Schießbefehl Noskes vom März 1919 hatte, aber nicht mit ihm identisch war. ${ }^{45}$ In Italien wiederum kam es anders: In keinem Moment nach dem Krieg nutzte der Staat Gewalt zur Untermauerung seiner Autori-

\footnotetext{
40 O.V.: Der Bürgerkrieg in Berlin, in: Vorwärts, Nr. 130 vom 12.3.1919 (Morgenausgabe).

41 Die Zahl der 75 gefallenen Freikorpssoldaten stammt aus einer zwar offiziellen Quelle der Zwischenkriegszeit, sollte aber mit Vorsicht genossen werden: Kriegsgeschichtlichen Forschungsanstalt des Heeres (Hrsg.): Die Wirren in der Hauptstadt und im nördlichen Deutschland 1918-1920 (Darstellungen aus den Nachkriegskämpfen deutscher Truppen und Freikorps, Bd. 6), Berlin 1940, S. 103.

42 O.V.: Die Opfer, in: Die Freiheit, Nr. 123 vom 14.3.1919 (Abendausgabe).

43 O.V.: Die Zahl der Toten, in: Germania, Nr. 110 vom 10.3.1919 (Abendausgabe); O.V.: Totenschau, in: Vorwärts, Nr. 126 vom 10.3.1919 (Morgenausgabe).

44 Tombs, Robert: The Paris Commune (Turning points), London 1999, S. $173 \mathrm{f}$.

45 Ylikangas, Heikki: Der Weg nach Tampere. Die Niederlage der Roten im finnischen Bürgerkrieg 1918, Berlin 2002; Horne/ Gerwarth, War in Peace; Bodo, Béla: Militia Violence and State Power in Hungary 1919-1922, in: Hungarian Studies Review 33 (2006), S. 121-156, Prusin, Alexander V.: The Lands Between. Conflict in the East European Borderlands 1870-1992 (zones of violence), Oxford 2010, S. 72 98; Gingeras, Ryan: Sorrowful Shores. Violence, Ethnicity, and the End of the Ottoman Empire $1912-$ 1923 (Oxford studies in modern european history), Oxford 2009. Zur »order to execute» des Irischen Freistaats: Foster, Roy: Modern Ireland 1600 - 1972, London 1988, S. 512 f.
} 
tät. Obwohl das Königreich siegreich aus dem Krieg hervorgegangen war, scheiterte das »liberale» Italien an der ausgebliebenen Demonstration seines Machtmonopols und hörte, vier Jahre nach Kriegsende, als Konsequenz daraus auf, zu existieren. ${ }^{46}$

Im Kontrast zu der optimistischen Idee, der Wiederaufbau Deutschlands könne friedlich und geordnet ablaufen, war die deutsche Politik im Frühling und Frühsommer 1919 durch Gewalt dominiert. Zu dieser Zeit war dies bereits ein Streitpunkt, der die deutsche politische Kultur wie kein anderes Thema spaltete. Im Laufe der Zeit entstand hierdurch ein Opfermythos innerhalb der deutschen Linken, welcher dazu beitrug, die Opposition gegen die Sozialdemokraten durch die gesamte Weimarer Republik hindurch zu stärken. ${ }^{47}$ Grundsätzlich verhinderte die Intensität und Häufigkeit der Gewalt die Entstehung eines stärkeren positiven Gründungsmythos: Anlässlich des 10. Jahrestages waren wenige Gruppen bereit, die Errungenschaften der Novemberrevolution zu feiern. Auf lange Sicht verhalf die kulturelle Symbolik, die die staatliche Gewalt im Zuge der Staatsgründung anklagte, antirepublikanischen Tendenzen, die die Staatsgründung mit Gewalt und Chaos gleichsetzten, zu immer größerer Bedeutung. Dies heißt aber nicht, dass die neue Republik von Beginn an zum Scheitern verurteilt war. ${ }^{48}$ Tatsächlich hatte die Weimarer Republik im Vergleich zur europäischen Politik der Zwischenkriegszeit eine beachtliche Stabilität vorzuweisen: Die Weimarer Demokratie überdauerte eine Reihe anderer Staaten, die in Folge des Ersten Weltkriegs gegründet worden waren. ${ }^{49}$ Es ist paradox: Ein Staat, dessen Gründung mit der beispiellosen Zurschaustellung von Gewalt einherging, wurde zu einem Staat, der unfähig war, seine eigene längerfristige Existenz durch Anwendung von staatlicher Gewalt zu sichern.

46 Eine exzellente Aufsatzsammlung zu »Italien nach dem Ersten Weltkrieg» ist zu finden bei Isnenghi, Mario/ Albanese, Giulia (Hrsg.): Gli italiani in guerra. Conflitti, identità, memorie dal Risorgimento ai nostril giorni Vol. 4 part I: Dall'impresa di Fiume alla seconda guerra mondiale 1919-1940, Turin 2008.

47 Dazu die historische Sichtweise der KP in: Internationaler Arbeiter-Verlag (Hrsg.): Illustrierte Geschichte der Deutschen Revolution, Berlin 1929.

48 Zur neuen Geschichtsschreibung über die Weimarer Republik: Fritzsche, Peter: Did Weimar Fail, in: The Journal of Modern History 68/3 (1996), S. 629-656; Ziemann, Benjamin: Weimar was Weimar: Politics, Culture and the Emplotment of the German Republic, in: German History 28/4 (2010), S. 542 571. Weiterführend Gallus, Alexander (Hrsg.): Die vergessene Revolution von 1918/19, Göttingen 2010; Ziemann, Benjamin: Contested Commemoration. Republican War Veterans and Weimar Political Culture, Cambridge 2013.

49 Gerwarth, Robert (Hrsg.): Twisted Paths. Europe 1914-1945, Oxford 2007. 